\title{
2PBC J0658.0-1746: a hard X-ray eclipsing polar in the orbital period gap
}

\author{
F. Bernardini,,${ }^{1,2,3 \star}$ D. de Martino, ${ }^{2}$ K. Mukai, ${ }^{4,5}$ M. Falanga,,${ }^{6,7}$ N. Masetti,, 9 \\ ${ }^{1}$ INAF - Osservatorio Astronomico di Roma, via Frascati 33, I-00040 Monteporzio Catone, Roma, Italy \\ 2 INAF - Osservatorio Astronomico di Capodimonte, Salita Moiariello 16, I-80131 Napoli, Italy \\ 3 New York University Abu Dhabi, Saadiyat Island, Abu Dhabi, 129188, United Arab Emirates \\ ${ }^{4}$ CRESST and X-Ray Astrophysics Laboratory, NASA Goddard Space Flight Center, Greenbelt, MD 20771, USA \\ 5 Department of Physics, University of Maryland, Baltimore County, 1000 Hilltop Circle, Baltimore, MD 21250, USA \\ 6 International Space Science Institute (ISSI), Hallerstrasse 6, 3012 Bern, Switzerland \\ 7 International Space Science Institute Beijing, No.1 Nanertiao, Zhongguancun, Haidian District, 100190 Beijing, China \\ ${ }^{8}$ INAF - Osservatorio di Astrofisica e Scienza dello Spazio, Via Gobetti 93/3, I-40129, Bologna, Italy \\ 9 Departamento de Ciencias Físicas, Universidad Andrés Bello, Fernández Concha 700, Las Condes, Santiago, Chile
}

Accepted XXX. Received YYY; in original form ZZZ

\begin{abstract}
The hard X-ray source 2PBC J0658.0-1746 was proposed as an eclipsing magnetic cataclysmic variable of the polar type, based on optical follow-ups. We present the first spectral and timing analysis at X-ray energies with XMM-Newton, complemented with archival X-ray, optical, IR photometry and spectroscopy. The X-ray emission shows bright and faint phases and total eclipses recurring every $2.38 \mathrm{~h}$, consistent with optical properties. This firmly identifies 2PBC J0658.0-1746 as an eclipsing polar, the second hard X-ray selected in the orbital period gap. The X-ray orbital modulation changes from cycle-to-cycle and the X-ray flux is strongly variable over the years, implying a non-stationary mass accretion rate both on short and long timescales. The X-ray eclipses allow to refine the orbital ephemeris with period 0.09913398(4)d, and to constrain the binary inclination $79^{\circ} \lesssim i \lesssim 90^{\circ}$ and the mass ratio $0.18<$ $\mathrm{M}_{2} / \mathrm{M}_{\mathrm{WD}}<0.40$. A companion mass $\mathrm{M}_{2}=0.2-0.25 \mathrm{M}_{\odot}$ with a radius $\mathrm{R}_{2}=0.24-$ $0.26 \mathrm{R}_{\odot}$ and spectral type $\sim \mathrm{M} 4$, at $\mathrm{D}=209_{-2}^{+3} \mathrm{pc}$, is derived. A lower limit to the white dwarf mass of $\sim 0.6 \mathrm{M}_{\odot}$ is obtained from the X-ray spectrum. An upper limit to the magnetic colatitude, $\beta \lesssim 50^{\circ}$, and a shift in azimuth, $\psi \sim 14^{\circ}$, of the main accreting pole are also estimated. The optical/IR spectral energy distribution shows large excess in the mid-IR due to lower harmonics of cyclotron emission. A highstate mass accretion rate $\sim 0.4-1 \times 10^{-10} \mathrm{M}_{\odot} \mathrm{yr}^{-1}$, lower than that of cataclysmic variables above the gap and close to that of systems below it, is estimated. With 2PBC J0658.0-1746, the number of hard X-ray selected polars increases to thirteen members, suggesting that they are not as rare as previously believed.
\end{abstract}

Key words: Novae, cataclysmic variables - white dwarfs - X-rays: individual: SWIFT J0658.0-1746 (aka 2PBC J0658.0-1746, 1RXS J065806.3-1744, 2MASS 06580591-1744249, WISE J065805.87-174424.5 )

\section{INTRODUCTION}

AM Herculis stars or polars, are magnetic cataclysmic variables (mCVs), short orbital period (up to a few hours) binary systems, hosting a strongly magnetic (10-230 MG) white dwarf (WD) primary that accretes matter via Roche lobe overflow from a main sequence late-type secondary (see Cropper 1990; Ferrario et al. 2015). The magnetic field is

^ E-mail:federico.bernardini@inaf.it strong enough to synchronise or quasi-synchronise the WD rotation at the binary orbital period $\left(\mathrm{P}_{\text {orb }}=\mathrm{P}_{\Omega}\right)$ so that material lost from the companion directly proceeds in a streamlike flow along the field lines from the inner Lagrangian point (L1) all the way down to the polar magnetic cap(s) on the WD surface, preventing the formation of an accretion disk. In the magnetically confined accretion flow, matter reaches supersonic velocities and a stand-off shock forms close to the WD surface (Aizu 1973). The post shock region (PSR) is hot $(\mathrm{kT} \sim 10-50 \mathrm{keV})$ and matter below cools down emitting thermal Bremsstrahlung (hard X-rays), and cyclotron 
radiation, emerging in the optical/near-infrared (nIR) band (Wu et al. 1994; Cropper et al. 1999). The efficiency of the two mechanisms depends on the magnetic field strength and local mass accretion rate, with the higher magnetic field systems being cyclotron dominated. Due to reprocessing of the hard X-rays and cyclotron radiation at the WD surface or heating from blobby accretion (König et al. 2006), an optically thick (blackbody-like) soft X-ray component can be observed. This was believed to be almost ubiquitous in polars during the ROSAT era. However, more recent observations with $X M M$-Newton showed an increasing number of such sources without a distinct soft X-ray component (see e.g. Ramsay \& Cropper 2004; Bernardini et al. 2014, 2017).

The X-ray (and frequently also optical) light curves of polars show periodic variability at the WD spin/orbital period, a clear signature of magnetic accretion. Their shape is extremely peculiar, usually showing a double peak with a broad, V-shaped dip due to partial obscuration of the emitting spot by the accretion stream (e.g. Ramsay et al. 2004; Bernardini et al. 2014). On top of short-term (hours) periodic variations, polars also show long-term (monthsyears) variability with high and low accretion states. Magnetic spots on the donor surface could temporarily fill the Lagrangian point (L1, Livio \& Pringle 1994) producing variable mass-transfer rates from the secondary (e.g. Hessman et al. 2000).

Polars represent the major subclass of $\mathrm{mCVs}$, with 130 systems identified so far (Ritter \& Kolb 2003; Ferrario et al. 2015) ${ }^{1}$, mainly discovered in the soft Xrays through the ROSAT (Schwope et al. 2002) and XMMNewton (e.g. Ramsay et al. 2009) serendipity surveys and in optical photometric surveys such as the Sloan Digital Sky Survey (SDSS; e.g Schmidt et al. 2008) or the Catalina Real-time Transient Survey (CRTS; e.g. Breedt et al. 2014). The lower-field (B $<10-20 \mathrm{MG}$ ) asynchronous systems, the so-called intermediate polars, amount to about 70 systems. Thus, mCVs represent about $20-25 \%$ of the whole CV class. The high incidence of magnetism among WDs in CVs, compared to $\sim 6-10 \%$ of isolated magnetic WDs (Ferrario et al. 2015), would either imply CV formation is favoured by magnetism or CV production enhances magnetism (Tout et al. 2008). Moreover, to explain their lower mass accretion rates with respect to non-magnetic CVs, it was proposed that mCVs, especially the polars, would suffer of reduced efficiency of the magnetic braking mechanism, operating as angular momentum loss (AML) above the $2-3 \mathrm{~h}$ orbital period gap, due to the strong coupling of the WD and companion magnetic fields (Wickramasinghe \& Wu 1994; Wickramasinghe \& Ferrario 2000; Ferrario et al. 2015).

Only recently, thanks to the hard X-ray surveys conducted by INTEGRAL with IBIS-ISGRI (Bird et al. 2016) and by the Neil Gehrels Swift observatory (hereafter Swift) with BAT (Oh et al. 2018), the number of discovered mCVs has substantially increased, representing $\sim 25 \%$ of galactic hard X-ray sources. While the majority are found to be of the intermediate polar-type, likely due to the dominance of Bremsstrahlung as main cooling mechanism, the number of polars is slowly, but steadily increasing amounting

1 For (Ritter \& Kolb 2003), see catalog version 7.24, more details at https://wwwmpa.mpa-garching.mpg.de/RKcat/. (excluding this work) to twelve systems (Bernardini et al. 2014; Mukai 2017; Bernardini et al. 2017), indicating that hard X-ray polars are not so rare as previously thought. Whether IPs only or also the polars are contributors to the low-luminosity population of Galactic X-ray sources is still under debate (Reis et al. 2013; Pretorius \& Mukai 2014).

Here is reported, in the framework of an identification programme with the XMM-Newton satellite of hard X-ray selected CV candidates (see e.g. Bernardini et al. 2012, 2013, 2017, 2019, and references therein), the first X-ray study of SWIFT J0658.0-1746 (hereafter J0658), discovered as an unidentified source in the Swift/BAT survey (Cusumano et al. 2014; Oh et al. 2018). It was proposed as a $\mathrm{mCV}$ because of its optical spectral characteristics (Rojas et al. 2017), until follow-up optical photometery in 2017-2018 revealed that it is an eclipsing system with a period of 0.0991370(3) days and large orbital modulation characteristic of polars (Halpern et al. 2018). The XMM-Newton observation, carried out a few months later in 2018, is here reported together with archival Swift/XRT and Swift/BAT light curves and spectra. These confirm the $2.38 \mathrm{~h}$ orbital period and that J0658 is a hard X-ray eclipsing polar. It is thus the $13^{\text {th }}$ hard X-ray discovered so far and the $2^{\text {nd }}$ falling in the middle of the $2-3 \mathrm{~h}$ orbital period gap, together with SWIFT J2218.4+1925 (Bernardini et al. 2014, and reference therein).

In Section 2 we report the observations and data reduction procedures, in Section 3 the timing and spectral analysis, in Section 4 we discuss the physical properties of this system in the context of mCVs, and in Section 5 we highlight our main conclusions.

\section{OBSERVATION AND DATA REDUCTION}

\section{$2.1 \quad X M M-N e w t o n$ observations}

J0658 has been observed for $\sim 33 \mathrm{ks}$ on 2018 September 19 by XMM-Newton with the European Photo Imaging Cameras (EPIC: PN, MOS1 and MOS2 Strüder et al. 2001; Turner et al. 2001; den Herder et al. 2001) as main instruments, complemented with simultaneous Optical Monitor (OM; Mason et al. 2001) V-band photometry and RGS spectroscopy (Strüder et al. 2001). The latter data, due to the faintness of the source, were of poor $\mathrm{S} / \mathrm{N}$ for a useful analysis. The details of the observation are reported in Table 1. Data were processed using the Science Analysis Software (SAS) version 17.0.0 and the latest calibration files available in 2019 May.

Source photon event lists and spectra for EPIC cameras were extracted from a circular region of radius $30 \mathrm{arcsec}$. The background was extracted in the same CCD where the target lies. The observation did not contain epochs dominated by particle background, so the whole exposure was used for the analysis.

Background-subtracted PN and MOSs light curves were produced with the task EPICLCCORR in the whole $0.3-12 \mathrm{keV}$ energy band, and in several energy sub-bands. The event arrival times were corrected to the Solar System barycentre by using the task BARYCEN. Before fitting, spectra were rebinned using SPECGROUP. A minimum of 30 and 25 counts in each bin for PN and MOSs, respectively, and a maximum 
oversampling of the energy resolution by a factor of three were set. Three time resolved spectra were also extracted, corresponding to the two highest bright phases, two less intense bright phases, and all faint phases of the spin/orbital cycle present in the exposure, respectively (see Section 3.1 and 3.2). The response matrix and the ancillary files were generated using the tasks RMFGEN and ARFGEN, respectively. $\mathrm{PN}$ and MOSs spectra were fitted together by using XsPEC version 12.10.1f package (Arnaud 1996).

The OM was operated in fast window mode using the V-band (5100-5800 $\AA$ ) filter simultaneously to the EPIC cameras. 10 exposure of $\sim 2.9 \mathrm{ks}$ each were performed. The background subtracted light curve was generated with the task OMFCHAIN with a bin time of $100 \mathrm{~s}$ and then the Solar System barycentric correction was applied.

\subsection{The Swift observations}

J0658 was observed twice by Swift/XRT (Burrows et al. 2005) a first time in 2009 February $(1.1 \mathrm{ks})$ and a second time in 2013 June $(1.6 \mathrm{ks})$. XRT $(0.3-10 \mathrm{keV})$ light curve and spectra were also extracted using the products generator available at Leicester Swift Science Centre (Evans et al. 2009). Since the source flux and spectral shape are consistent during the two epochs, we produced and analysed a single averaged spectrum.

Swift/BAT (Barthelmy et al. 2005) has built up an allsky map of the hard X-ray sky (14-195 keV), thanks to its wide field of view. The eight-channel spectra (14-195 $\mathrm{keV}$ ), response file, and light curve from the first 105 months of BAT monitoring (Oh et al. 2018) were downloaded from the publicly available archive at the NASA's Goddard Space Flight Center website ${ }^{2}$. We restricted the spectral analysis to $\mathrm{E}<80 \mathrm{keV}$.

\section{DATA ANALYSIS AND RESULTS}

\section{$3.1 \quad$ Timing analysis}

First the background subtracted Swift/BAT lightcurve of J0658 was inspected and found to show clear long-term (years) variability, with a broad ( $F W H M \sim 5$ months) peak centered at $\mathrm{t} \sim 90$ months (2012 May). The $14-195 \mathrm{keV}$ flux at the peak is $\sim 7 \times 10^{-11} \mathrm{erg} \mathrm{cm}^{-2} \mathrm{~s}^{-1}$, while the average flux over $\sim 7$ yrs outside the peak is $\sim 4.5 \times 10^{-12}$ $\mathrm{erg} \mathrm{cm} \mathrm{cm}^{-2} \mathrm{~s}^{-1}$ (factor $\sim 16$ lower). The Swift/XRT, 0.3-10 keV, flux level in $2009 / 2013$ was $1.8 \times 10^{-11} \mathrm{erg} \mathrm{cm}^{-2} \mathrm{~s}^{-1}$ assuming a simple power-law spectral model (Section 3.2).

The average magnitude during the $\mathrm{OM}$ pointing is $\mathrm{V}=$ $18.5 \pm 0.2 \mathrm{mag}$, which is $\sim 2.2 \mathrm{mag}$ fainter than what obtained interpolating the $\mathrm{B}$ and $\mathrm{R}$ magnitudes (Halpern et al. 2018) from the USNO B1.0 catalog (Monet et al. 2003), that were taken over several decades. J0658 is found 1.3 mag fainter with respect to the USNO B1.0 catalog also in the V-band (17.2 mag), when using the Gaia DR2 G-band magnitude and red ( $\mathrm{Rp}$ ) and blue (Bp) colors (Gaia Collaboration et al. $2018)^{3}$ along with the color-transformation by Evans et al.

2 https://swift.gsfc.nasa.gov/results/bs105mon/

3 The Gaia DR2 data covers a 22 months period, starting from July 2014.
(2018). Furthermore, when later observed in December 2017 (Halpern et al. 2018) the R-band photometry was consistent with the R-band magnitude obtained from the Gaia color-transformation (R 16.7). J0658 WAS also detected in the Sloan Digital Sky Survey (SDSS) DR13 (Blanton et al. 2017) and observed in 2008 at $\mathrm{g}=17.2$, similarly to the Gaia level. All this indicates that J0658 faded after DecemberFebruary 2018 and is highly variable on long timescales.

The $0.3-12 \mathrm{keV}$ background subtracted PN light curve (Figure 1) shows a periodic modulation with a structured bright phase and a faint phase which are typical of polars (four cycles are covered). The bright phase is characterised by a total eclipse that separates two unequal peaks. The modulation changes in shape from cycle to cycle, with the first and last bright phases (hereafter B) brighter than the other two (b). The count rate during the faint phases (f) is above zero. The power spectrum, computed in the $0.3-12$ $\mathrm{keV}$ range, shows a strong peak at $\sim 10^{-4} \mathrm{~Hz}$ together with several harmonics up to the 7th. A first estimate of the period of the fundamental harmonic is obtained by simply fitting the EPIC averaged (PN, MOS1, and MOS2) $0.3-12 \mathrm{keV}$ light curve with a series of sinusoids consisting of the fundamental plus the first 6 harmonics and gives $P=8565 \pm 34$ s. This is perfectly consistent with that derived from optical photometry (Halpern et al. 2018). All uncertainties are hereafter at $1 \sigma$ confidence level, if not otherwise specified.

The V-band OM light curve does not show clear signs of bright and faint phases and so a detectable orbital modulation, but the eclipses (only three covered) are clearly observed (Figure 1), where $V \sim 19.4 \pm 0.4$ mag. Halpern et al. (2018) found instead that in 2017 December, the light curve of J0658 shows a periodic modulation with a double-peaked maximum at $\mathrm{R} \sim 15.5 \mathrm{mag}$ rising from a baseline of $\mathrm{R} \sim 17.3$ mag. All this indicates that in 2018 September J0658 was in a low-intermediate state.

\subsubsection{The eclipse}

The coverage of four eclipses in the X-ray band allows us to derive a new, improved, orbital ephemeris. The times of eclipse centres were measured using a Gaussian fit and crosschecked by manual inspection on the 50s-binned background subtracted PN light curve. The ingress(egress) times were measured from two points that have a flux that is half of the average value immediately before(after) the eclipse. The X-ray eclipse has $100 \%$ depth (count rates going to zero) and all four measures have consistent length with an average value of $10.73 \pm 0.43 \mathrm{~min}$. The total length of the eclipse (time of first to the fourth/last contact) is $13.89 \pm 0.06 \mathrm{~min}$ for the first and third eclipse and slightly longer, $14.8 \pm 0.1 \mathrm{~min}$, in the second and last one. The OM V-band eclipses, as measured on the 100s-binned light curve, last $9.64 \pm 0.48 \mathrm{~min}$ (second to third contact) and $13.6 \pm 0.6 \mathrm{~min}$ (first to last contact). The $\mathrm{X}$-ray and optical eclipses lengths are consistent within their uncertainties and with the previous optical observations by Halpern et al. (2018). Instead, the drop in $\mathrm{V}$ magnitude with respect to the average out-of-eclipse level, is $\sim 1 \mathrm{mag}$, much smaller than previously observed. In order to obtain an improved ephemeris, the nine eclipse egresses reported 
Table 1. Summary of main observation parameters for all instruments. Uncertainties are at $1 \sigma$ confidence level.

\begin{tabular}{cccccccc}
\hline $\begin{array}{c}\text { Source Name } \\
\text { Coordinates (J2000) }\end{array}$ & Telescope & OBSID & Instrument & $\begin{array}{c}\text { Date } \\
\text { yyyy-mm-dd }\end{array}$ & $\begin{array}{c}\mathrm{UT}_{\text {start }} \\
\text { hh:mm }\end{array}$ & $\begin{array}{c}\mathrm{T}_{\exp }{ }^{a} \\
\mathrm{ks}\end{array}$ & $\begin{array}{c}\text { Net Source Count Rate } \\
\mathrm{c} / \mathrm{s}\end{array}$ \\
\hline SWIFT J0658.0-1746 & XMM-Newton & 0820330701 & EPIC-PN $^{c}$ & $2018-09-19$ & $18: 29$ & 32.9 & $0.093 \pm 0.002$ \\
& & & EPIC-MOS1 $^{c}$ & $2018-09-19$ & $18: 21$ & 33.3 & $0.023 \pm 0.001$ \\
RA=06:58:05.873 & & & EPIC-MOS2 $^{c}$ & $2018-09-19$ & $18: 21$ & 33.3 & $0.025 \pm 0.001$ \\
Dec=-17:44:24.40 & & & OM-V $^{d}$ & $2018-09-19$ & $18: 26$ & 29.0 & $18.5 \pm 0.2^{e}$ \\
& Swift & \multirow{2}{*}{038850001} & XRT & $2009-02-13$ & $05: 30$ & 1.1 & $0.24 \pm 0.01$ \\
& Swift & 047443001 & XRT $^{f}$ & $2013-06-07$ & $02: 27$ & 1.6 & $0.21 \pm 0.01$ \\
& Swift & & BAT & & & 185500 & $1.4 \pm 0.3 \times 10^{-5}$ \\
\hline
\end{tabular}

$a$ Net exposure time. The PN ontime is $23 \mathrm{ks}$ only.

$b$ Coordinates of the optical counterpart.

c Small window mode (medium filter applied for MOS1 and MOS2, thin filter applied for PN).

$d$ Fast image mode. The central wavelength of the $\mathrm{V}$ filter is $5430 \AA$.

$e$ OM V magnitude in the Vega system.

$f$ This pointing consists of two PC mode snapshots of $0.8 \mathrm{ks}$ each, the second starting on 2013-06-08 at 08:55.

$g$ All available pointings collected from 2004 December to 2013 September are summed together.

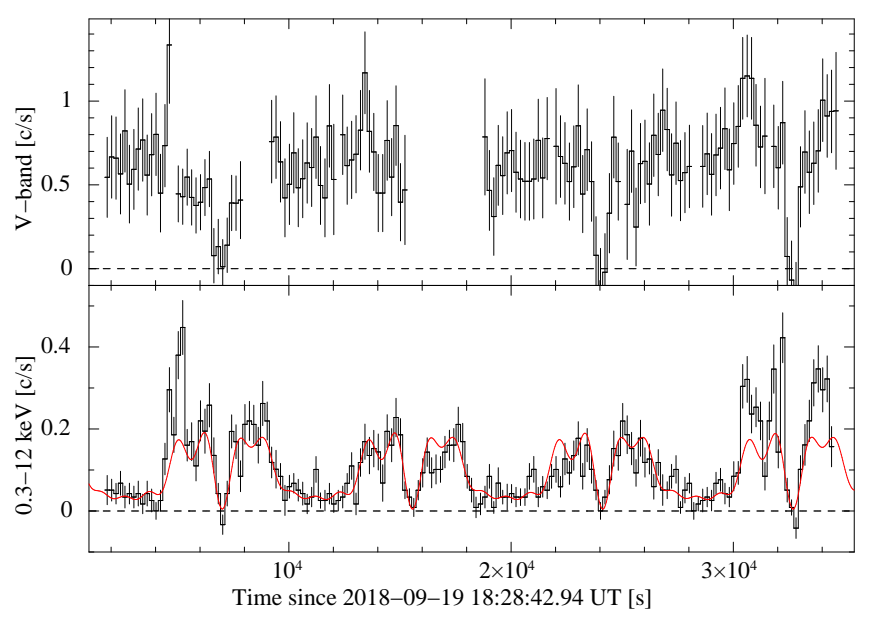

Figure 1. 2018 September 19 OM V-band (upper panel) and PN 0.3-12 keV (lower panel) background subtracted light curves binned at $200 \mathrm{~s}$, where for plotting purposes is also shown the result of a fit made with 7 sinusoids, the orbital period (8565 s) and its first six harmonics (red curve).

by Halpern et al. (2018) ${ }^{4}$ and the four times of X-ray eclipse egress were used. The OM V-band eclipses are not used since the light curve has a lower $\mathrm{S} / \mathrm{N}$. A linear regression using the thirteen measures gives: $\mathrm{T}^{\text {egress }}(\mathrm{BJD})=2458381.333958(65)$ and $\mathrm{P}_{\Omega}=0.09913398(4) \mathrm{d}$. The observed-minus-calculated residuals were inspected against trends finding that a constant period gives an acceptable fit. The excursions on average are within $20 \mathrm{~s}$ around zero, with only one within $34 \mathrm{~s}$ (Figure 2).

The background-subtracted (PN, MOS1, and MOS2) light curves in the $0.3-12 \mathrm{keV}$ and $\mathrm{V}$-band were then folded with the improved ephemeris. Since the source shows cycle-

${ }^{4}$ We did not use the times of ingress since only eight are reported by Halpern et al. (2018)

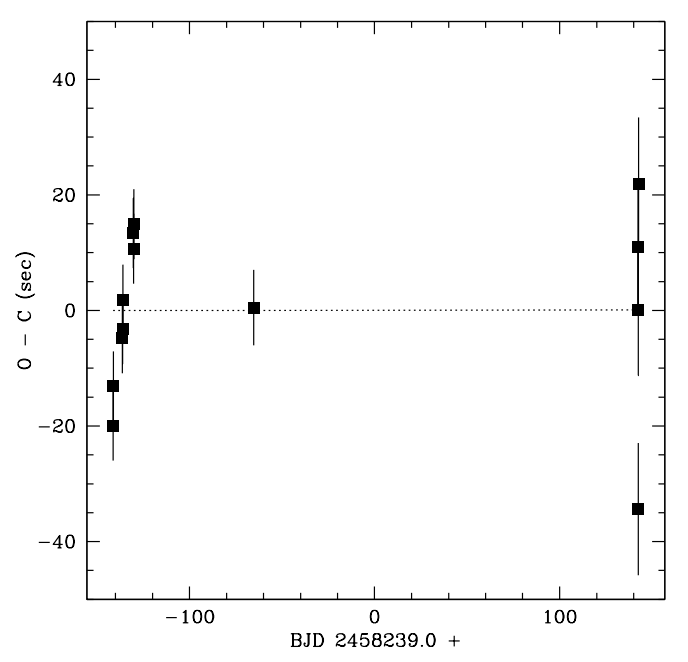

Figure 2. Deviations of the times of eclipse egress from the linear ephemeris (dotted line) obtained by fitting the nine optical and the four X-ray egresses (Section 3.1.1).

to-cycle variability the folding was performed on two intervals encompassing the two strongest bright phases (B) and the two less intense (b) ${ }^{5}$. In Figure 3 the X-ray and V-band folded orbital light curves are shown. Notably is the presence in the X-ray orbital modulation during $\mathrm{B}$ of a dip preceding the eclipse (at $\phi_{\Omega} \sim 0.82$ ), not seen during b. The pulsed fraction $(\mathrm{PF})^{6}$ of the fundamental harmonic (excluding the eclipse interval) in the X-rays is $57 \pm 7 \%$ during $\mathrm{B}$. In the optical the $\mathrm{PF}$ is consistent with zero, and only the eclipse is clearly present. The second, b, interval is characterised by a

5 More specifically interval B extends between MJD 58380.771 and 58380.875 and between MJD 58381.079 and 58381.550, whilst interval b extends between MJD 58380.884 and 58381.088.

6 The pulsed fraction is here defined as the semi-amplitude of the modulation divided by its average value. 

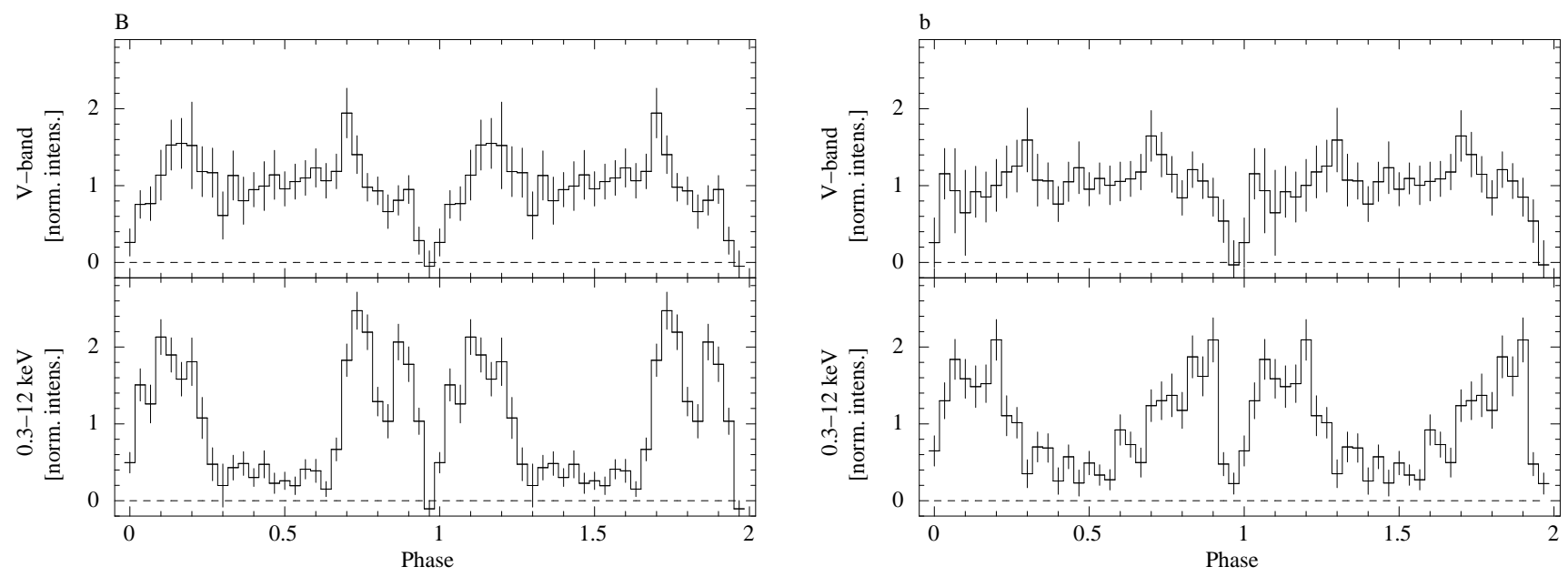

Figure 3. Left: Background-subtracted V-band (top) and PN 0.3-12 keV (bottom) light curves, folded using the improved ephemeris (Table 4) for the interval covering the two stronger bright phases (B). Two cycles are shown for plotting purposes. Right: The same as left panel, but for the interval covering the two less intense bright phases (b). Note the presence of a dip before the X-ray eclipse, only during $\mathrm{B}$, at $\phi \sim 0.82$.

bright phase with no clear presence of a dip in both bands. The orbital modulation in the $\mathrm{X}$-ray band $\mathrm{PF}=54 \pm 7 \%$ and consistent with zero in the optical band. Inspection of hardness ratios folded at the orbital period in the two intervals do not show any variability, neither at the dip feature, likely because of the low $\mathrm{S} / \mathrm{N}$. These findings could indicate that the source has a highly variable accretion rate on timescales of hours and when brighter the accretion stream passing through the line-of-sight of the observer partially obscures the accreting region.

\subsection{Spectral analysis}

The broad-band (0.3-80 keV), time-averaged, XMM-Newton EPIC plus BAT combined, spectrum was first fitted using an optically thin plasma (CEMEK in XSPEC), a model that accounts for the run of emission measure with temperature in PSR of mCVs, (see e.g. Mukai 2017; Bernardini et al. 2018). While this model well fits the data, the inter-calibration constant, used to account for instrument calibration discrepancies and spectral variability, is extremely large, 60 for the BAT spectrum. This indicates that J0658, when observed by XMM-Newton, is much fainter than the BAT average. This is consistent with the observed low optical level of the source. Moreover, the combined spectra do not provide substantial improvement in the fit and particularly the maximum temperature of the CEMEK model. We then here report the results from the fits using the $0.3-10 \mathrm{keV}$ spectra of the three EPIC cameras.

We linked all models parameters among different spectra, leaving free to vary only the cross-normalization constant (fixed to 1 for the PN). We obtained statistically acceptable fits (Table 2, and Figure 4) using both CEMEK $\left(\chi_{v}^{2}=1.04,94\right.$ d.o.f $)$ and a model consisting of two MEKALS, absorbed only by a total covering column TBAB $\left(\chi_{v}^{2}=1.03\right.$, 93 d.o.f.). The absorber accounts for the absorption from the Galactic interstellar medium. Metal abundances $\left(\mathrm{A}_{\mathrm{Z}}\right)$ with respect to Solar were set to that of (Wilms et al. 2000) and left free to vary. Due to the source faintness (and low $\mathrm{S} / \mathrm{N}$ ), the fluorescent $6.4 \mathrm{keV}$ Fe emission line, usually observed in mCVs, is not detected in J0658. Moreover, neither a partial covering absorbing column, usually present in these accreting systems due to self-obscuration from the accretion stream, is needed to fit the data. This in turn explains why the PF does not change with the energy interval. The emission measure in CEMEK follows a power-law in temperature $d E M=\left(T / T_{\max }\right)^{a-1} d T / T_{\max }$, where $\mathrm{T}_{\max }$ can be considered as a lower limit to the real shock temperature. The best fit gives $\mathrm{kT}_{\max }=19 \pm_{5}^{10} \mathrm{keV}$ and $\alpha=0.56 \pm_{0.23}^{0.19}$. The model with two mekals (cold and hot, respectively) gives instead $\mathrm{kT}_{c}=0.66 \pm 0.04 \mathrm{keV}$ and $\mathrm{kT}_{h}=6.1 \pm_{0.6}^{0.9} \mathrm{keV}$. The total absorber column density is a factor of about 10 lower than the Galactic value in the direction of the source, $3.4-3.7 \times 10^{21} \mathrm{~cm}^{-2}$ (Dickey \& Lockman 1990; Kalberla et al. 2005 ), consistent with a close-by source (see Sect 4 ). The fits also do not require a black-body component, adding this source to the increasing number of polars without a distinct soft component ${ }^{7}$.

The $0.3-10 \mathrm{keV}$ spectra of interval $\mathrm{B}$, b, and $\mathrm{f}$, were also analysed. The spectra of the three EPIC cameras, for each interval separately, were fitted simultaneously using the best-fitting average spectral models by linking all the model components with the exception of $\mathrm{N}_{\mathrm{H}_{\text {Tbab }}}$ and $\mathrm{A}_{\mathrm{Z}}$ that were set to their average spectral best fitting values. For what concerns the CEMEK model, first the spectrum of interval $\mathrm{B}$ was fitted with both $\mathrm{kT}_{\max }$ and $\alpha$ left free to vary, but they resulted poorly constrained: $\mathrm{kT}_{\text {max }}=22.6 \pm_{8}^{24} \mathrm{keV}, \alpha=$ $0.63 \pm 0.20$, and $n=6 \pm 1 \times 10^{-4}\left(\chi_{v}^{2}=1.08,54\right.$ d.o.f. $)$. Then, $\alpha$ was set to its average spectrum best-fitting value. The fits show that $\mathrm{kT}_{\max }$ increases (the source gets harder) from a minimum of $10.3 \pm_{2.5}^{3.7} \mathrm{keV}$ to a maximum of $27.4 \pm_{4.3}^{5.4} \mathrm{keV}$ (the same does the normalization of CEMEK) as the X-ray

7 Adding a blackbody with $\mathrm{kT}=0.05 \mathrm{keV}$ (fixed), we derive a $3 \sigma$ upper limit to the $0.3-10 \mathrm{keV}$ flux, which is $<8 \times 10^{-14} \mathrm{erg} \mathrm{cm}^{-2} \mathrm{~s}^{-1}$. 
flux increases (Table 3). In the case of the model made with two MEKALS, instead, the temperature of the two optically thin plasma remain constant within uncertainty, but their normalizations increase with the flux. In particular that of the hot component significantly increases from a minimum of $0.5 \pm 0.1 \times 10^{-4}$ to a maximum of $3.3 \pm 0.2 \times 10^{-4}$.

Given the source long-term X-ray variability, the XRT spectrum averaged over the pointings in 2009 and 2013 was also analysed. First the broad-band spectrum, including BAT, was fitted using the same models in Table 2. The inter-calibration constant for BAT is $\sim 0.20$, implying that J0658 when observed by XRT is brighter than the BAT average. Moreover, $\mathrm{kT}_{\max }$ is unconstrained, reaching the model maximum value $(100 \mathrm{keV})^{8}$. This also suggests that J0658 spectrum is very hard when observed by XRT. The XRT spectrum was then fitted alone, first using the same models in Table 2, and $\mathrm{kT}_{\max }$ (or $\mathrm{kT}_{h}$ ) is found to be unconstrained ${ }^{9}$. Then, a simple power-law model was used and gives: $\mathrm{N}_{h}=0.05$ (fixed), $\Gamma=0.9 \pm 0.1$, norm $=1.0 \pm 0.1 \times 10^{-3}$, $\mathrm{F}_{0.3-10 \mathrm{keV}}=1.8 \pm 0.2 \times 10^{-11}\left(\chi_{v}^{2}=0.6211\right.$ d.o.f.. $)$. This shows that J0658, when observed by XRT, is a factor of $\sim 60$ brighter than in 2018. It also shows that it is much harder $\left(<\Gamma_{2018}>=1.74 \pm 0.04\right)$, confirming the hardening trend with increasing X-ray fluxes (Figure 4).

\section{DISCUSSION}

The X-ray observation of J0658 with the detection of the $\mathrm{X}$-ray eclipses allowed to refine the orbital ephemeris and enabled to constrain the system parameters and to obtain information on the magnetic field geometry of the accreting WD (Table 4).

\subsection{Binary system parameters}

The time elapsed from the first to the 3rd contact (or from the 2 nd to the 4 th contact) corresponds to $\Delta \phi=0.087 \pm 0.008$. This allows to place constrains on the donor star radius in units of binary separation: $\left(R_{2} / a\right)^{2}=\left(\sin ^{2}(\pi \Delta \phi)+\right.$ $\cos ^{2}(\pi \Delta \phi) \cos ^{2}(i)$ (Horne et al. 1982), where $\mathrm{R}_{2}$ is the donor star radius, $a$ is the binary separation, and $i$ is the binary inclination. Since J0658 is found to be accreting, the secondary star should be filling its Roche-lobe. This equation, together with the equivalent Roche lobe radius approximation by Eggleton (1983), defines an unique relation between the binary inclination $i$ and the mass ratio $\mathrm{q}=\mathrm{M}_{2} / \mathrm{M}_{1}$. In Figure 5 the observed eclipse length (blue line) is reported together with its uncertainties (red lines). Different eclipse fractions allow for a limited range of $\mathrm{q}-\mathrm{i}$ values (black lines). To constrain the $q-i$ values, the density of a secondary star filling its Roche-lobe was used: $<\rho>=3 \mathrm{M}_{2} / 4 \pi \mathrm{R}_{2}^{2}=3 \pi / 0.459^{3} \mathrm{GP}_{\Omega}^{2}$ (Faulkner et al. 1972), which allows to put constrains on the radius of the secondary star for a given mass. Then, to get an estimate of the donor mass and radius, the donor star mass-radius (M$\mathrm{R}$ ) relation adopting the $2.38 \mathrm{~h}$ orbital period is compared

\footnotetext{
8 Using PWAB (normally adopted when CV spectra look unusually hard using other simpler absorption model), instead of TBAB, $\mathrm{kT}_{\text {max }}$ remains unconstrained.

9 The same is true if PWAB is used.
}

with the M-R relations for late-type main sequence stars as derived from the evolutionary models by Baraffe et al. (2015) at 1 Gyr and 5 Gyr and for donors in CVs as derived from the semi-empirical sequence by Knigge et al. (2011). These are displayed in Figure 6. The points where the M-R relation intersects these sequences define the likely ranges for the donor mass and its radius: $\mathrm{M}_{2}=0.2-0.25 \mathrm{M}_{\odot}$ and $\mathrm{R}_{2}=\mathrm{R}_{\mathrm{L} 2}=0.24-0.26 \mathrm{R}_{\odot}$, respectively. Adopting as a lower limit to the shock temperature $\mathrm{kT}_{\max }$, as derived from the X-ray spectral fits of interval $\mathrm{B}$, using its $1 \sigma$ uncertainty (23-33 keV; Table 3, first row), a minimum WD mass in the range $0.62-0.76 \mathrm{M}_{\odot}$ is derived. Then, the mass ratio is limited to $q<0.4$, for $M_{W D}>0.6 \mathrm{M}_{\odot}$ and $\mathrm{M}_{2}=0.25 \mathrm{M}_{\odot}$. In the q-i plane (Figure 5), the eclipse fraction limits the binary inclination in the range $79^{\circ} \lesssim \mathrm{i} \lesssim 90^{\circ}$ and the mass ratio in the range $0.18<\mathrm{q}<0.40$ (shaded region). The latter, for a donor mass of $0.2-0.25 \mathrm{M}_{\odot}$, would give $0.5<\mathrm{M}_{\mathrm{WD}}<1.4 \mathrm{M}_{\odot}$. A massive WD is not favoured, as it would imply a shock temperature higher than $100 \mathrm{keV}$. We then conservatively adopt $\mathrm{M}_{\mathrm{WD}}=0.6-1.0 \mathrm{M}_{\odot}$. From the inferred inclination and donor radius, a binary orbital separation in the range $0.69-1.18 \mathrm{R}_{\odot}$ is derived.

\subsection{The X-ray emitting region}

The X-ray modulation reveals the typical bright and faint phases seen in the polars, which are produced by the accretion flow above the main (or upper) pole that comes into (bright phase) and out of (faint phase) view if the magnetic and rotation axes are offset by an angle $\beta$ defined as the magnetic colatitude (Cropper 1988). The non-zero count rate during the faint phase could suggest either that the accreting upper pole does not completely disappear behind the WD limb or that a second emitting region is present. The length of the faint phase and the derived inclination can be used to restrict the range of values of the magnetic colatitude of the main pole as $\cos i=\cos \left(\pi \Delta \phi_{\text {faint }}\right) \tan \beta$, (Cropper 1990). The X-ray faint phase is evaluated in interval $\mathrm{B}$, where the modulation is better defined, and is determined as the phase range where the count rate is below the average value: $\Delta \phi_{\text {faint }}=0.42 \pm 0.03$. Using $i=79-90^{\circ}$, the magnetic colatitude, neglecting any height and width of the column, is restricted to $\beta \lesssim 50^{\circ}$. Here we note that the colatitude is undetermined for $i=90^{\circ}$. $\beta$ could be larger if there is a vertical or horizontal extent of the emission region (e.g. Vogel et al. 2008).

For $\beta<90^{\circ}$, the main accreting pole is on the same side of the orbital plane as the line of sight of the observer. The azimuth $\psi$ is the angle from the line centres of the two stars to the main pole projected onto the orbital plane. This can be defined as $\psi=\phi_{\text {eclipse }}-\phi_{\text {max }}$,cent , where $\phi_{\text {eclipse }}$ is the phase of the eclipse centre and $\phi_{\text {max }}$,cent the phase of the centre of the maximum, i.e. when the accretion column is closest to the line-of-sight (Cropper 1988). We then derive $\psi \sim 14^{\circ}$.

The decline to the eclipses (1st to 2nd contact) is found to be different in interval $\mathrm{B}$ and $\mathrm{b}$. It ranges from $42-53 \mathrm{~s}$ during interval B, while it is longer (120-162 s) during b. The rise from the eclipses (3rd to 4th contact) is instead longer in B (2.3-3.7 min) than in b, where is only $44 \mathrm{~s}$ and $74 \mathrm{~s}$. The differences are likely due to the low $\mathrm{S} / \mathrm{N}$ of the data, preventing quantitative estimates of the size of the X-ray emitting region. 

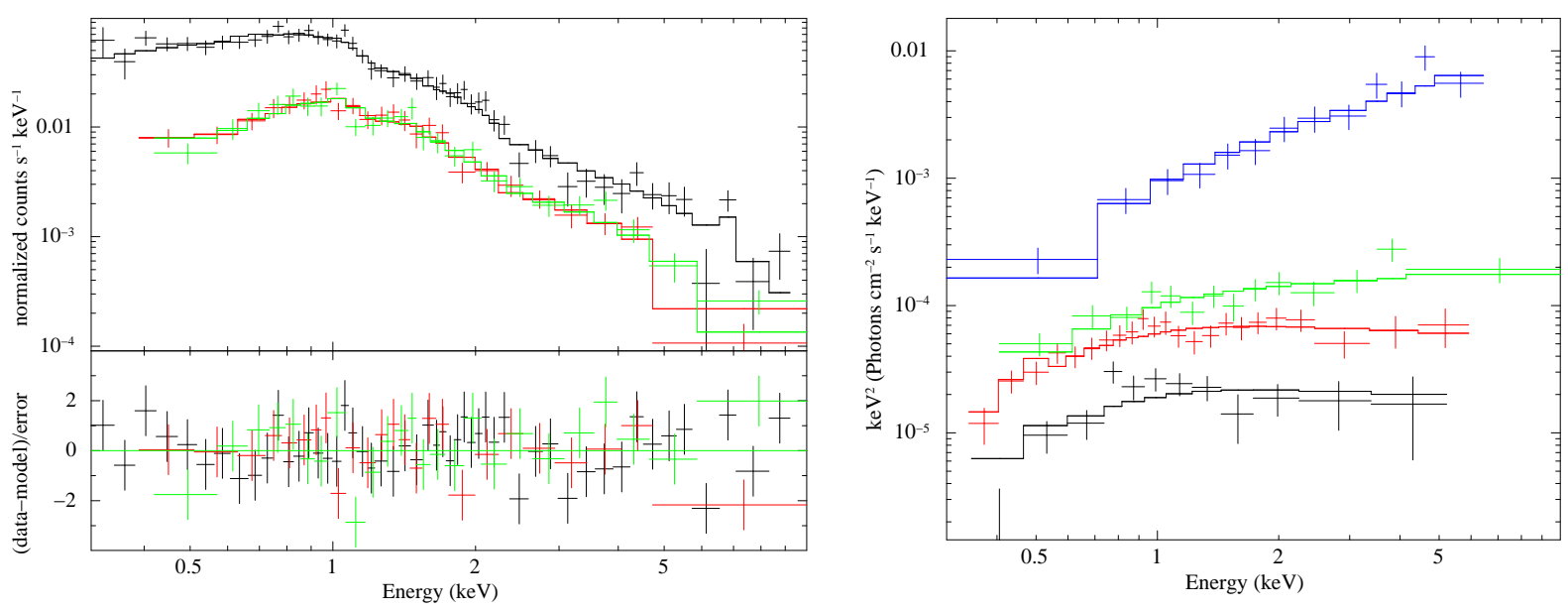

Figure 4. Left: $0.3-10 \mathrm{keV}$ count, averaged, spectrum fitted using CEMEK (Table 2). PN spectrum is in black, MOS1 in red, MOS2 in green. Residuals are shown in the lower panel. Right: Unfolded 0.3-10 keV spectra using a simple, phenomenological, power-law model. $\mathrm{XRT}$ spectrum is in blue, PN interval $\mathrm{B}$ in green, PN interval $\mathrm{b}$ in red, PN interval $\mathrm{f}$ in black. The spectra are plotted together to highlight the source flux variability, and spectral hardening, with increasing X-ray flux.

Table 2. Best fit models to the average $0.3-10 \mathrm{keV}$ spectrum. Uncertainties are at $1 \sigma$ confidence level. The unabsorbed $0.3-10 \mathrm{keV}$ fluxes are also reported.

\begin{tabular}{|c|c|c|c|c|c|c|c|c|c|}
\hline mod. & $\begin{array}{c}\mathrm{N}_{\mathrm{H}_{\text {Thab }}} \\
10^{22} \\
\mathrm{~cm}^{-2}\end{array}$ & $\begin{array}{l}\mathrm{kT}_{c} \\
\mathrm{keV}\end{array}$ & $\begin{array}{c}\mathrm{n} \\
10^{-4}\end{array}$ & $\begin{array}{c}\mathrm{kT}_{h}{ }^{a} \\
\mathrm{keV}\end{array}$ & $\begin{array}{c}\mathrm{n}_{h} \\
10^{-4}\end{array}$ & $\alpha$ & $\mathrm{A}_{\mathrm{Z}}$ & $\begin{array}{c}\mathrm{F}_{0.3-10} \\
10^{-13} \\
\mathrm{erg} / \mathrm{cm}^{2} / \mathrm{s}\end{array}$ & $\chi_{v}^{2} /$ dof \\
\hline cemek & $0.05 \pm 0.02$ & - & - & $19 \pm_{5}^{10}$ & $2.4 \pm 0.5$ & $0.56 \pm_{0.23}^{0.19}$ & $0.83 \pm_{0.32}^{0.43}$ & $3.1 \pm 0.6$ & $1.04 / 94$ \\
\hline $2 \mathrm{mek}$ & $0.027 \pm 0.013$ & $0.66 \pm 0.04$ & $0.14 \pm_{0.04}^{0.08}$ & $6.1 \pm_{0.6}^{0.9}$ & $1.5 \pm 0.1$ & -0.23 & $1.1 \pm 0.4$ & $3.0 \pm 0.3$ & $1.03 / 93$ \\
\hline
\end{tabular}

${ }^{a}$ For cemekl, it is the maximum temperature.

Table 3. Spectral parameters for the bright and faint phases, where interval $\mathrm{B}$ corresponds to the two stronger bright phases, $\mathrm{b}$ to the two weaker bright phases, and $\mathrm{f}$ to all faint phases (excluding the eclipse intervals). $\mathrm{N}_{\mathrm{H}_{\text {Tbab }}}$ and $\mathrm{A}_{\mathrm{Z}}$ are fixed to their average spectrum best-fitting values. The unabsorbed $0.3-10 \mathrm{keV}$ are also reported. Uncertainties are at $1 \sigma$ confidence level.

\begin{tabular}{|c|c|c|c|c|c|c|c|c|c|}
\hline $\begin{array}{l}\text { mod. } \\
\text { Interval }\end{array}$ & $\begin{array}{c}\mathrm{N}_{\mathrm{H}_{\text {Thab }}} \\
10^{22} \\
\mathrm{~cm}^{-2}\end{array}$ & $\begin{array}{l}\mathrm{kT}_{c} \\
\mathrm{keV}\end{array}$ & $\begin{array}{c}\mathrm{n}_{c} \\
10^{-4}\end{array}$ & $\begin{array}{c}\mathrm{kT}_{h}{ }^{a} \\
\mathrm{keV}\end{array}$ & $\begin{array}{c}\mathrm{n}_{\boldsymbol{h}} \\
10^{-4}\end{array}$ & $\alpha$ & $A_{Z}$ & $\begin{array}{c}\mathrm{F}_{0.3-10} \\
10^{-13} \\
\mathrm{erg} / \mathrm{cm}^{2} / \mathrm{s}\end{array}$ & $\chi_{v}^{2} /$ dof \\
\hline \multicolumn{10}{|l|}{ cemek $^{b}$} \\
\hline B & 0.05 & - & - & $27.4 \pm_{4.3}^{5.4}$ & $5.2 \pm 2.0$ & 0.56 & 0.83 & $7.1 \pm 0.3$ & $1.06 / 55$ \\
\hline $\mathrm{b}$ & 0.05 & - & - & $15.1 \pm_{2.3}^{\frac{4}{2}: 9}$ & $2.7 \pm 1.2$ & 0.56 & 0.83 & $3.5 \pm 0.2$ & $0.59 / 37$ \\
\hline $\mathrm{f}$ & 0.05 & - & - & $10.3 \pm_{2.5}^{3.7}$ & $0.8 \pm 0.1$ & 0.56 & 0.83 & $1.0 \pm 0.2$ & $0.98 / 17$ \\
\hline \multicolumn{10}{|l|}{$2 \mathrm{mek}$} \\
\hline B & 0.027 & $0.82 \pm 0.10$ & $0.27 \pm 0.07$ & $7.1 \pm_{1.2}^{1.7}$ & $3.3 \pm 0.2$ & - & 1.1 & $6.7 \pm 0.4$ & $1.08 / 53$ \\
\hline $\mathrm{b}$ & 0.027 & $0.67 \pm 0.10$ & $0.19 \pm 0.03$ & $5.7 \pm_{0.9}^{1.3}$ & $1.6 \pm 0.1$ & - & 1.1 & $3.3 \pm 0.3$ & $0.69 / 35$ \\
\hline $\mathrm{f}$ & 0.027 & $0.63 \pm 0.10$ & $0.08 \pm 0.02$ & $5.0 \pm 1.5$ & $0.5 \pm 0.1$ & - & 1.1 & $0.9 \pm 0.2$ & $0.96 / 15$ \\
\hline
\end{tabular}

${ }^{a}$ For cemekl, it is the maximum temperature.

${ }^{b}$ Also $\alpha$ is fixed to the average spectrum best-fitting value.

The spectral analysis shows that the PSR emitting region is highly variable over time. The X-ray flux of the bright phase changes by a factor of two from cycle to cycle, whilst the minimum flux is essentially constant. During the faint phase the flux is $~ 3-6$ times lower than that during the bright phases (interval $\mathrm{b}$ and $\mathrm{B}$, respectively). Although it is not possible to precisely assess the temperature structure of the PSR with present data and the adopted spectral model, the fact that lower temperatures are found during the bright phases of lower intensity (interval b) might suggest that the PSR is not stationary and could adjust itself according to the instantaneous mass accretion rate. 


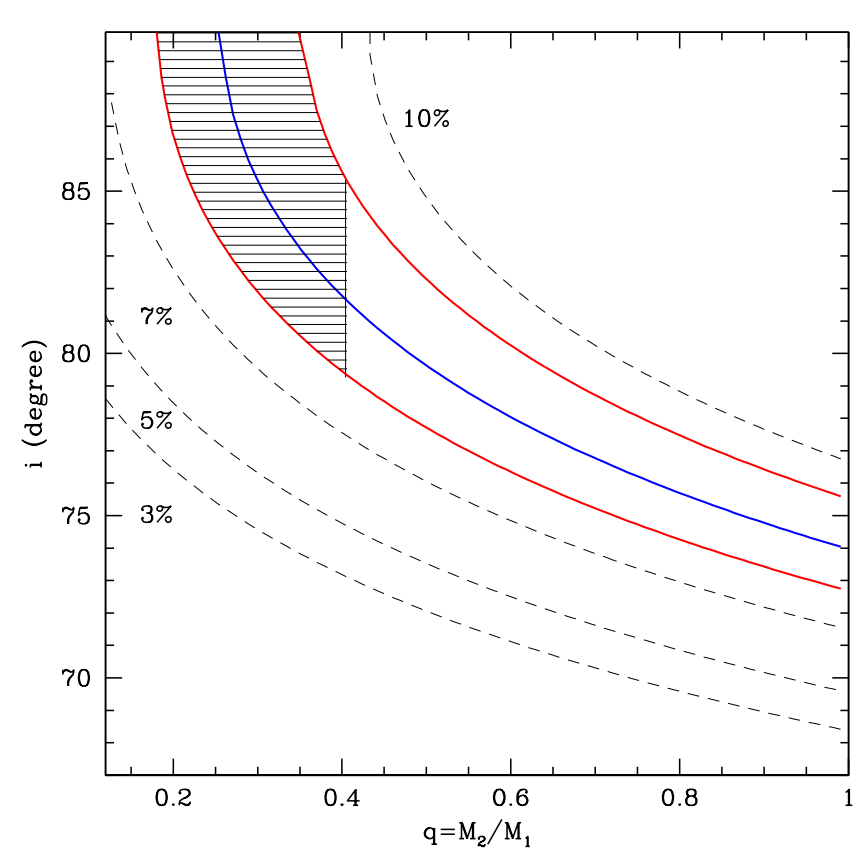

Figure 5. The eclipse fraction (its duration compared to the orbital period) as a function of the mass ratio and the orbital inclination. The blue and red lines indicate the range allowed by the measured eclipse fraction and uncertainties respectively. The dashed black lines indicate the inclinations versus mass ratio for different eclipse fractions. The shaded area corresponds to the limits set by the donor and primary masses (see Section 4.1 for details).

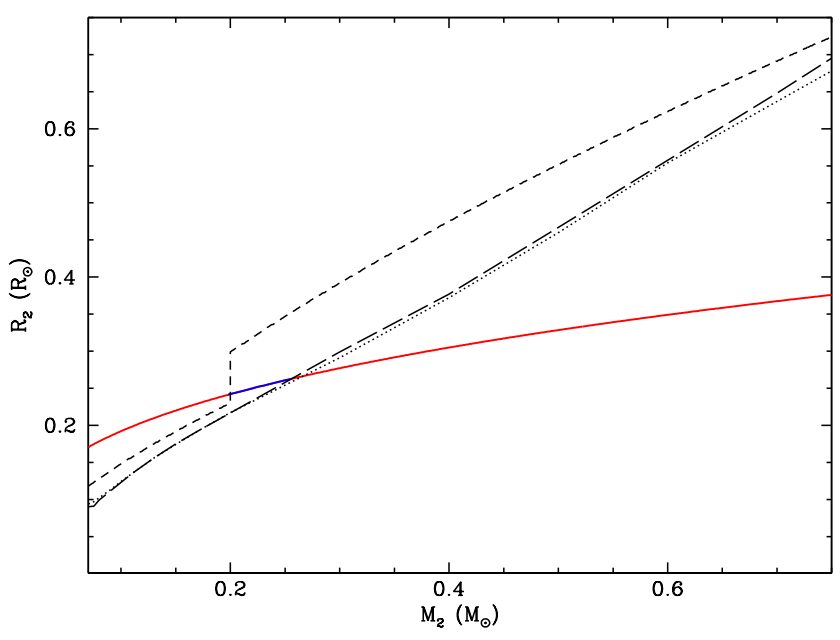

Figure 6. The M-R relation adopting a Roche-lobe filling secondary star and a binary orbital period of $2.38 \mathrm{~h}$ (red line). The $\mathrm{M}-\mathrm{R}$ relation for low-mass main sequence stars derived from the evolutionary models of (Baraffe et al. 2015) at 1 $\dot{G} y r$ (dotted line) and 5 Gyr (long-dashed line) and from the semi-empirical evolutionary sequence for CV donors by (Knigge et al. 2011) (shortdashed line). The points of intersection with the sequences give the likely range (blue line) of the secondary star parameters.

\subsection{The accretion and stellar components}

J0658 has been observed by Gaia (Gaia Collaboration et al. 2018) that in DR2 has provided a precise parallax of $\pi=$ $4.75 \pm 0.05$ mas, accurate at $\sim 1 \%$. With this accuracy the direct inversion is well justified giving $\mathrm{D}=211 \pm 2 \mathrm{pc}$. We however also derive the distance to the source using the weak distance prior that varies as a function of Galactic longitude and latitude according to the Galactic model described in (Bailer-Jones et al. 2018) ${ }^{10}$ and find $\mathrm{D}=209_{-2}^{+3} \mathrm{pc}$ and a scale length $\mathrm{L}=1573 \mathrm{pc}$. We adopt the latter as best estimate of the distance since it is independent of assumptions on physical properties of individual stars. This close distance may explain the detection of this source in hard X-rays, similarly to the case of the other twelve polars that are also found within $500 \mathrm{pc}$.

The extinction in the optical band, as derived from the hydrogen column density $\mathrm{N}_{\mathrm{H}}$ of the $\mathrm{X}$-ray spectral fits, is $\mathrm{E}(\mathrm{B}-\mathrm{V})=0.08$. For a donor mass $\mathrm{M}_{2}=0.2-0.25 \mathrm{M}_{\odot}$ and radius $R_{2}=0.24-0.26 R_{\odot}$ both the CV semi-empirical sequence (Knigge et al. 2011) and the low-mass evolutionary models (Baraffe et al. 2015) predict a M4 spectral type with effective temperature of $\sim 3300 \mathrm{~K}$. The expected absolute magnitude in the V-band is 12.3-12.4 mag for both models, which in turn predicts an apparent extincted V magnitude of 19.3-19.4 mag, fully consistent with the observed magnitude $(19.4 \pm 0.3)$ during the eclipse. The R-band magnitude during the eclipse is $18.5 \mathrm{mag}$ (Halpern et al. 2018). Again, using $\mathrm{E}(\mathrm{B}-\mathrm{V})$, the distance and predicted absolute magnitude $\mathrm{R}=11.4-11.6 \mathrm{mag}$, the expected apparent $\mathrm{R}$-band magnitude is indeed 18.2-18.4 mag. All this confirms the donor star parameters derived above.

The epoch of the XMM-Newton observation, due to the lower accretion rate, is more favourable to get a constrain on the direct emission from the WD atmosphere. Note that effective temperatures of WDs in polars have been found systematically lower than those in non-magnetic CVs (Araujo-Betancor et al. 2005), with values ranging from $10 \mathrm{kK}$ to $20 \mathrm{kK}$ and for $\mathrm{P}_{\Omega}<3 \mathrm{~h}$, up to $\sim 14 \mathrm{kK}$. The lack of colour photometry and/or spectroscopy allows to only derive an upper limit to the WD effective temperature. For this purpose we consider $\mathrm{M}_{W D}=0.6-1.0 \mathrm{M}_{\odot}$ and $\mathrm{R}_{W D}=5.5-8.5 \times 10^{8} \mathrm{~cm}$, using the WD mass-radius relation of Nauenberg (1972). We use the DA WD model atmospheres by Koester (2010), adopting $\log g=8.0$ and varying the effective temperatures in the range $10-24 \mathrm{kK}$, and scale the spectral distributions (SED) using the range for the WD radius and the distance to the source reported above. We then set the upper limit considering that the WD photospheric emission must be lower than the observed V-band flux outside the eclipse, that gives $\mathrm{T}_{W D}<12-22 \mathrm{kK}$ (Figure 7$)$.

J0658 is also detected at near-IR ( J, H, and K) wavelengths in the 2MASS survey (Skrutskie et al. 2006) as 2MASS J06580591-1744249, as well as in the four (W1, W2, W3 and W4) bands in the WISE survey (Cutri \& et al. 2013) as WISE J065805.87-174424.5. We then built a broadband SED from 0.35 to $22 \mu$ using the SDSS u, g, r, i, and $\mathrm{z}$ photometry and also include the optical spectrum from Rojas et al. (2017) (2011 April 20), the OM V and 
R-band measures and the 2MASS and WISE catalogue magnitudes (all measures are corrected for extinction with $\mathrm{E}(\mathrm{B}-\mathrm{V})=0.08)$, keeping in mind that they uncover different epochs (1998 for 2MASS, 2008 for SDSS and 2010 for WISE). The SED is displayed in Figure 7. Since J0658 has additionally been observed by the reactivated WISE mission $^{11}$ between April 3, 2014 and October 12, 2018, the single $7.7 \mathrm{~s}$ exposure data in the two $\mathrm{W} 1(3.4 \mu)$ and $\mathrm{W} 2(4.6 \mu)$ bands were retrieved. When folded at the refined ephemeris (Section 3.1.1, Table 4), the NEOWISE photometry clearly shows eclipses at these wavelengths (Figure 8), where the drop in magnitude is $\Delta \mathrm{W} 1 \sim 1.0 \mathrm{mag}$ and $\Delta \mathrm{W} 2 \sim 1.2 \mathrm{mag}$. We are unable to further improve the orbital period with these data since the eclipse is covered only in a few epochs with a handful of exposures. The out-of-eclipse phases are characterised by a large variability, likely due to the multiepoch coverage of the observations and thus not used in the SED. The eclipse measures in the V, R and W1, W2 bands are also shown in Figure 7. A comparison with the predicted broad-band fluxes from a $0.2 \mathrm{M}_{\odot}$ donor at $3000 \mathrm{~K}$ from (Baraffe et al. 2015) clearly shows the agreement with all the eclipse fluxes. Instead, the fluxes out-of-eclipse show an excess extending down to $22 \mu$. The excess at optical wavelengths could be a mixture of the accretion stream and cyclotron contributions, while the latter could be dominant in the nIR. Indeed, Harrison \& Campbell (2015) showed that those polars with strong nIR excess (down to $\sim 12-22 \mu$ ) are dominated by the fundamental and/or lower harmonics. In particular those systems having the fundamental in the longer-wavelength WISE band (W3) should be low-field (B < 10 MG) polars. We note that J0658 could be a low-field polar.

To evaluate the excess of flux, we subtracted from the observed out-of-eclipse fluxes in each band the contribution of the WD or donor star. More specifically for the Sloan $u$ and $\mathrm{g}$ filters we subtracted the model atmosphere flux of a WD at $22 \mathrm{kK}$ and of $1.0 \mathrm{M}_{\odot}$ here assumed as an upper limit, while for the longer wavelength bands we subtracted the predicted fluxes for a donor of $0.2 \mathrm{M}_{\odot}$ and $3300 \mathrm{~K}$. Here we do not include the V-band data of September 2018, since J0658 was in a faint state. This gives an integrated flux of $\sim 1.2 \times 10^{-11} \mathrm{erg} \mathrm{cm}^{-2} \mathrm{~s}^{-1}$. If the optical spectrum from Rojas et al. (2017) is used, instead of the Sloan data, an integrated flux of $\sim 2.2 \times 10^{-11} \mathrm{erg} \mathrm{cm}^{-2} \mathrm{~s}^{-1}$ is obtained. The corresponding luminosity is $\sim 1.2 \times 10^{32} \mathrm{erg} \mathrm{s}^{-1}$. The latter could be regarded as an estimate of the high state accretion luminosity at optical/IR wavelengths. Comparison with the X-ray luminosity is then performed using the higher state Swift observations in 2009 and 2013 for which the derived flux (from 0.01 to $10 \mathrm{keV}$ ) is $\sim 7.8 \times 10^{-11} \mathrm{erg} \mathrm{cm}^{-2} \mathrm{~s}^{-1}$ obtained from the simple power-law fit (Sect.3.2), and the average BAT spectrum for which the derived (10-200 kev) flux using a simple power-law model $(\Gamma=2.0)$ is $\sim 1.1 \times 10^{-11} \mathrm{erg} \mathrm{cm}^{-2} \mathrm{~s}^{-1}$. The corresponding $0.01-200 \mathrm{keV}$ X-ray luminosity is then: $\sim 4.7 \times 10^{32} \mathrm{erg} \mathrm{s}^{-1}$, which is a factor of $\sim 4$ larger than that derived in the optical/IR range. We then estimate the mass accretion rate assuming $\mathrm{L}_{\mathrm{accr}}=\mathrm{L}_{\mathrm{x}}+\mathrm{L}_{\mathrm{opt}}=\mathrm{GM}_{\mathrm{WD}} \dot{\mathrm{M}} / \mathrm{R}_{\mathrm{WD}}$. For $\mathrm{M}_{\mathrm{WD}} \sim 0.6-1.0 \mathrm{M}_{\odot}$,

11 WISE spacecraft was reactivated in December 2013 and named NEOWISE, https://irsa.ipac.caltech.edu/Missions/wise.html.

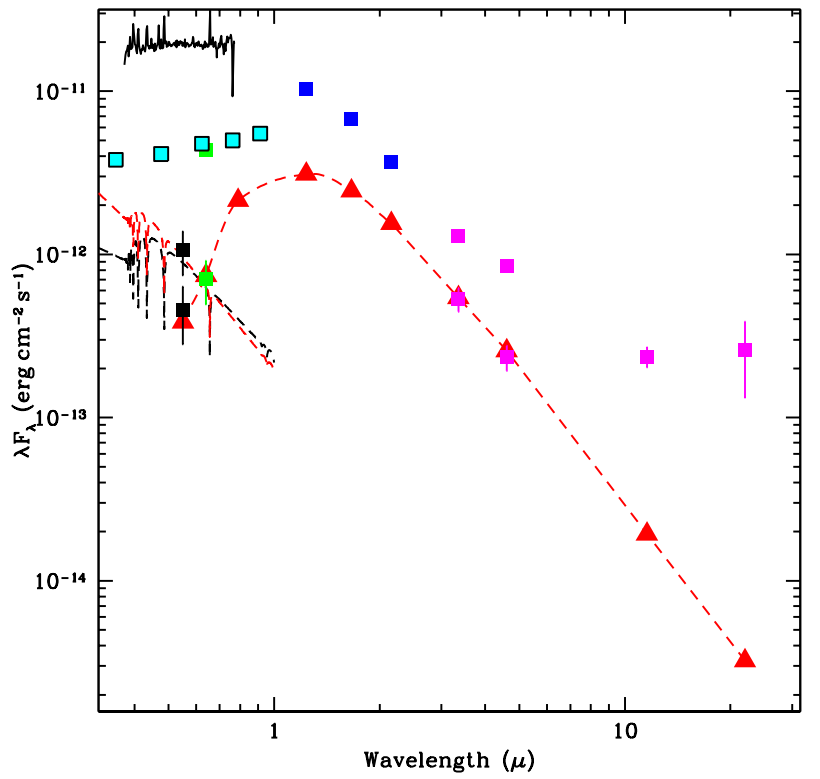

Figure 7. The multi-epoch SED of J0658 constructed using the OM V-band photometry (black points) during and outside the eclipse, together with the R-band fluxes (green points) during and outside the eclipse (Halpern et al. 2018) and the optical spectra (black curve; Rojas et al. 2017) or alternatively the SDSS (cyan squares) photometry (note the flux variability at different epochs), and the 2MASS (blue squares) and WISE photometry (magenta squares). The eclipse fluxes in the W1 and W2 bands are also reported in magenta. Overplotted as black and red dashed lines are the DA model atmospheres for a WD mass of 0.6 and $1.0 \mathrm{M}_{\odot}$ and temperatures of $12 \mathrm{kK}$ and $22 \mathrm{kK}$, respectively (Koester 2010). Red triangles are instead the predicted optical (VRI) and nIR (JHK) fluxes for a $0.2 \mathrm{M}_{\odot}$ and $\mathrm{T}_{2}=3300 \mathrm{~K}$ donor star (Baraffe et al. 2015) and the predicted WISE W1, W2, W3, and W4 fluxes for a main-sequence star at the same temperature (Pecaut \& Mamajek 2013), together with a dashed red line to help readability. While during the eclipse the fluxes well match those predicted by the late-type companion models, the out-of eclipse fluxes, show an excess (Section 4.3).

the total accretion luminosity of $\sim 5.9 \times 10^{32} \mathrm{erg} \mathrm{s}^{-1}$ gives $\dot{\mathrm{M}} \sim 3.8-9.6 \times 10^{-11} \mathrm{M}_{\odot} \mathrm{yr}^{-1}$. This value is lower than those of CVs above the $2-3 \mathrm{~h}$ orbital period gap and rather consistent with those of systems below it (Knigge et al. 2011). Considering that J0658 is located in the middle of the gap, the mass transfer rate is expected to drastically drop when entering the gap. CVs can be born inside the gap and contribute to the number of systems observed there (Goliasch \& Nelson 2015). Another possibility is that J0658 is one of the polars that never had an effective magnetic braking, and hence it evolved, without a significant break, from a longer initial period (Webbink \& Wickramasinghe 2002).

J0658 is then the second hard X-ray discovered polar in the orbital period gap, together with SWIFT J2218.4+1925 $(2.16 \mathrm{~h})$ (Bernardini et al. 2014). So far the total number of polars in the gap amounts to 34 systems (Ritter \& Kolb 2003, updated 2016 catalog), 26\% of the whole polar population. The lack of a well-defined gap in $\mathrm{mCVs}$ (Webbink \& Wickramasinghe 2002) was ascribed to the the 


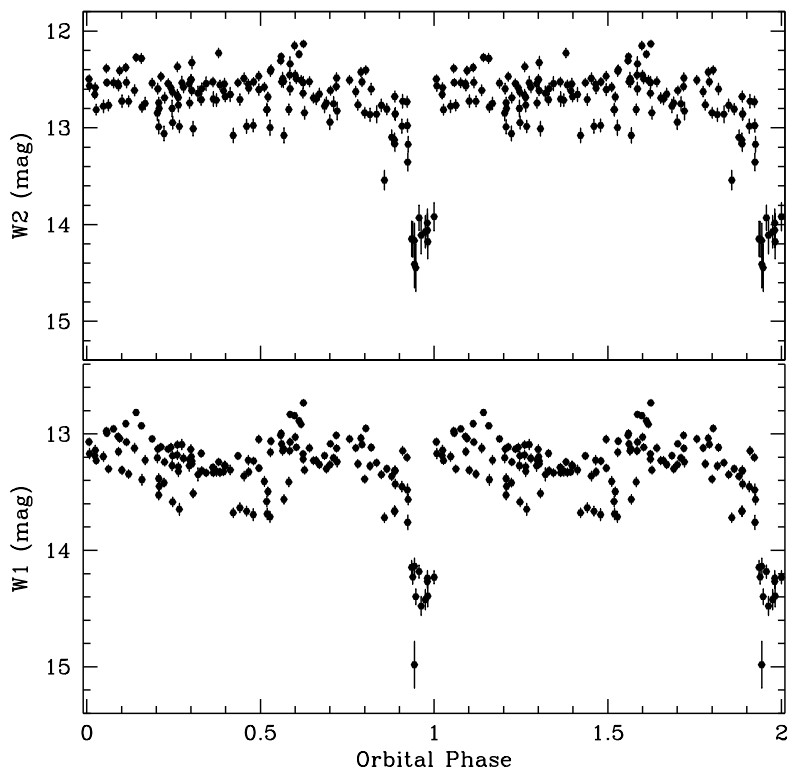

Figure 8. The NEOWISE (April 2014 - October 2018) folded light curves of J0658 in W1 $(3.4 \mu)$ and W2 $(4.6 \mu)$ bands at the $2.38 \mathrm{~h}$ orbital period displaying eclipses also in the nIR. The exposure for each point is $7.7 \mathrm{~s}$.

Table 4. Binary system parameters for J0658.

\begin{tabular}{lc}
\hline Orbital period & $0.09913398(4) \mathrm{d}$ \\
Eclipse egress (BJD) & $2458381.333958(65)$ \\
\hline Orbital inclination $(i)$ & $79-90^{\circ}$ \\
Mass Ratio (q) & $0.18-0.40$ \\
WD Mass ${ }^{\mathrm{a}}\left(\mathrm{M}_{\mathrm{WD}}\right)$ & $>0.6 \mathrm{M}_{\odot}$ \\
Secondary Mass $\left(\mathrm{M}_{2}\right)$ & $0.2-0.25 \mathrm{M}_{\odot}$ \\
Secondary Radius $\left(\mathrm{R}_{2}\right)$ & $0.24-0.26 \mathrm{R}_{\odot}$ \\
Secondary Spectral Type & $\mathrm{M} 4$ \\
Distance (D) & $209_{-2}^{+3} \mathrm{pc}$ \\
Main Pole colatitude $(\beta)$ & $\lesssim 50^{\circ}$ \\
Main Pole azimuth $(\psi)$ & $\sim 14^{\circ}$ \\
\hline
\end{tabular}

a This represents a lower limit to the real WD mass.

tight coupling between the donor and WD magnetic fields, reducing the magnetic braking as the wind from the secondary star may be trapped within the WD magnetosphere.

\section{CONCLUSIONS}

J0658 is the thirteen hard X-ray selected polar discovered so far. The presence of eclipses with a period of $2.38 \mathrm{~h}$ locates it in the middle of the $2-3 \mathrm{~h}$ orbital period $\mathrm{CV}$ gap. Its X-ray emission is modulated at the orbital period and the intensity of the modulation is variable (factor of two) from cycle to cycle, a signature of a non-stationary mass accretion rate. The X-ray luminosity it is also found to be highly variable on long (yrs) timescales (factor of sixty) and XMM-Newton caught it at the lowest state ever observed. The X-ray spec- trum is thermal and consistent with a multi-temperature structure, as observed in many magnetic systems.

The eclipses also allow to constrain the binary inclination between $79^{\circ}$ and $90^{\circ}$, the mass ratio $\mathrm{q}=0.18-0.4$ and orbital separation $\mathrm{a}=0.69-1.18 \mathrm{R}_{\odot}$. From Gaia parallax the distance is firmly set at $209 \pm_{2}^{3}$ pc. We estimate a donor star mass of $0.2-0.25 \mathrm{M}_{\odot}$ assumed to fill its Rochelobe at a temperature of $3000 \mathrm{~K}$ and a lower limit to the WD mass of $0.6 \mathrm{M}_{\odot}$, with an upper limit to its effective temperature of $12-22 \mathrm{kK}$. The presence of a non-negligible optical/IR excess, suggests a contribution of cyclotron radiation at these wavelengths. J0658 may host a weakly magnetic WD (B $\lesssim 10 \mathrm{MG})$. We tentatively estimate a magnetic field orientation of the main accreting pole with colatitude $\beta \lesssim 50^{\circ}$ and an azimuth of $\sim 14^{\circ}$. Spectropolarimetry will be crucial to determine the magnetic field strength and its topology for this polar. With thirteen systems found as hard X-ray polars, the possibility that this sub-class of $\mathrm{mCV}$ could be a non-negligible contributor to the hard X-ray lowluminosity Galactic population $\left(\lesssim 10^{32} \mathrm{erg} \mathrm{s}^{-1}\right)$ should be further investigated.

\section{ACKNOWLEDGEMENTS}

FB is founded by the European Union's Horizon 2020 research and innovation programme under the Marie Sklodowska-Curie grant agreement n. 664931. DdM and NM acknowledge financial support from the Italian Space Agency and National Institute for Astrophysics, ASI/INAF, under agreements ASI-INAF I/037/12/0 and ASI-INAF n.2017-14-H.0 and from INAF SKA/CTA Presidential Decree N. 70/2016. This work is based on observations obtained with $X M M-N e w t o n$, an ESA science mission with instruments and contributions directly funded by ESA Member States, with Swift, a NASA science mission with Italian participation, and with Gaia, a ESA mission. Gaia data are processed by the Data Processing and Analysis Consortium (DPAC). We thank the anonymous referee for the useful comments. We acknowledge useful discussion with Carlo Ferrigno.

\section{REFERENCES}

Aizu K., 1973, Prog. Theor. Phys., 49, 1184

Araujo-Betancor S., Gänsicke B. T., Long K. S., Beuermann K., de Martino D., Sion E. M., Szkody P., 2005, ApJ, 622, 589

Arnaud K. A., 1996, in Jacoby G. H., Barnes J., eds, Astronomical Society of the Pacific Conference Series Vol. 101, Astronomical Data Analysis Software and Systems V. p. 17

Bailer-Jones C. A. L., Rybizki J., Fouesneau M., Mantelet G., Andrae R., 2018, AJ, 156, 58

Baraffe I., Homeier D., Allard F., Chabrier G., 2015, A\&A, 577, A42

Barthelmy S. D., et al., 2005, Space Sci. Rev., 120, 143

Bernardini F., de Martino D., Falanga M., Mukai K., Matt G., Bonnet-Bidaud J.-M., Masetti N., Mouchet M., 2012, A\&A, 542, A22

Bernardini F., et al., 2013, MNRAS, 435, 2822

Bernardini F., de Martino D., Mukai K., Falanga M., 2014, MNRAS, 445, 1403 
Bernardini F., de Martino D., Mukai K., Russell D. M., Falanga M., Masetti N., Ferrigno C., Israel G., 2017, MNRAS, 470,4815

Bernardini F., de Martino D., Mukai K., Falanga M., 2018, MNRAS, 478, 1185

Bernardini F., de Martino D., Mukai K., Falanga M., 2019, MNRAS, 484, 101

Bird A. J., et al., 2016, ApJS, 223, 15

Blanton M. R., et al., 2017, AJ, 154, 28

Breedt E., et al., 2014, MNRAS, 443, 3174

Burrows D. N., et al., 2005, Space Sci. Rev., 120, 165

Cropper M., 1988, MNRAS, 231, 597

Cropper M., 1990, SSRv, 54, 195

Cropper M., Wu K., Ramsay G., Kocabiyik A., 1999, MNRAS, 306,684

Cusumano G., Segreto A., La Parola V., Maselli A., 2014, in Proceedings of Swift: 10 Years of Discovery (SWIFT 10), held 2-5 December 2014 at La Sapienza University, Rome, Italy. id.132. p. 132

Cutri R. M., et al. 2013, VizieR Online Data Catalog, 2328

Dickey J. M., Lockman F. J., 1990, ARA\&A, 28, 215

Eggleton P. P., 1983, ApJ, 268, 368

Evans P. A., Beardmore A., Osborne J., O'Brian P., Willingale R. andStarling R., Burrows D. e. a., 2009, MNRAS, 397, 1177

Evans D. W., et al., 2018, A\&A, 616, A4

Faulkner J., Flannery B. P., Warner B., 1972, ApJ, 175, L79

Ferrario L., de Martino D., Gänsicke B. T., 2015, Space Sci. Rev., 191, 111

Gaia Collaboration Brown A. G. A., Vallenari A., Prusti T., de Bruijne J. H. J., Babusiaux C., Bailer-Jones C. A. L., 2018, preprint, (arXiv:1804.09365)

Goliasch J., Nelson L., 2015, ApJ, 809, 80

Halpern J. P., Thorstensen J. R., Cho P., Collver G., Motsoaledi M., Breytenbach H., Buckley D. A. H., Woudt P. A., 2018, AJ, 155, 247

Harrison T. E., Campbell R. K., 2015, ApJS, 219, 32

Hessman F. V., Gänsicke B. T., Mattei J. A., 2000, A\&A, 361, 952

Horne K., Gomer R. H., Lanning H. H., 1982, ApJ, 252, 681

Kalberla P. M. W., Burton W. B., Hartmann D., Arnal E. M., Bajaja E., Morras R., Pöppel W. G. L., 2005, A\&A, 440, 775

Knigge C., Baraffe I., Patterson J., 2011, ApJS, 194, 28

Koester D., 2010, Mem. Soc. Astron. Italiana, 81, 921

König M., Beuermann K., Gänsicke B. T., 2006, A\&A, 449, 1129

Livio M., Pringle J. E., 1994, ApJ, 427, 956

Mason K. O., et al., 2001, A\&A, 365, L36

Monet D. G., et al., 2003, AJ, 125, 984

Mukai K., 2017, PASP, 129, 062001

Nauenberg M., 1972, ApJ, 175, 417

Oh K., et al., 2018, ApJS, 235, 4

Pecaut M. J., Mamajek E. E., 2013, ApJS, 208, 9

Pretorius M. L., Mukai K., 2014, MNRAS, 442, 2580

Ramsay G., Cropper M., 2004, MNRAS, 347, 497

Ramsay G., Bridge C., Cropper M., Mason K., Cordova F., Priedhorsky W., 2004, MNRAS, 354, 773

Ramsay G., Rosen S., Hakala P., Barclay T., 2009, MNRAS, 395, 416

Reis R., Wheatley P., Gänsicke B., Osborne J., 2013, MNRAS, 430, 1994

Ritter H., Kolb U., 2003, A\&A, 404, 301

Rojas A. F., et al., 2017, A\&A, 602, A124

Schmidt G. D., Smith P. S., Szkody P., Anderson S. F., 2008, PASP, 120,160

Schwope A. D., Brunner H., Buckley D., Greiner J., Heyden K. v. d., Neizvestny S., Potter S., Schwarz R., 2002, A\&A, 396, 895

Skrutskie M. F., et al., 2006, AJ, 131, 1163

Strüder L., et al., 2001, A\&A, 365, L18
Tout C. A., Wickramasinghe D. T., Liebert J., Ferrario L., Pringle J. E., 2008, MNRAS, 387, 897

Turner M. J. L., et al., 2001, A\&A, 365, L27

Vogel J., Byckling K., Schwope A., Osborne J., Schwarz R., Watson M., 2008, A\&A, 485, 787

Webbink R., Wickramasinghe D., 2002, MNRAS, 335, 1

Wickramasinghe D. T., Ferrario L., 2000, PASP, 112, 873

Wickramasinghe D. T., Wu K., 1994, MNRAS, 266, L1

Wilms J., Allen A., McCray R., 2000, ApJ, 542, 914

Wu K., Chanmugam G., Shaviv G., 1994, ApJ, 426, 664

den Herder J. W., et al., 2001, A\&A, 365, L7

This paper has been typeset from a $\mathrm{T}_{\mathrm{E}} \mathrm{X} / \mathrm{LAT}_{\mathrm{E}} \mathrm{X}$ file prepared by the author. 